\title{
Reseña
}

\section{Innovación educativa en Iberoamérica: estudio de casos de investigación}

http://dx.doi.org/10.18566/comunica.n43.a07

Fecha de recepción: 28 de octubre de 2020

Fecha de aceptación: 13 de noviembre de 2020

El libro Innovación educativa en Iberoamérica: estudio de casos de investigación, de Óscar Boude Figueredo y Erika Jaillier, presenta un llamado a la comunidad de investigadores y docentes en tecnología educativa de Iberoamérica a transformar sus prácticas pedagógicas, pues de continuar así, tal y como lo presenta Oppenheimer en su texto Sálvese quien pueda, los docentes podrían llegar a ser reemplazados por robots.

Bajo esta problemática, los autores muestran una alternativa de solución a la comunidad académica a través de la compilación de 14 casos de innovación educativa realizados en Iberoamérica, que pueden ser utilizados por los profesores e investigadores en tecnología educativa para identificar la ruta en pro de transformar sus prácticas pedagógicas en escenarios formativos que respondan a las dinámicas y necesidades de la sociedad digital.

El primer capítulo presenta una investigación que tenía como objetivo develar las bases epistemológicas del modelo pedagógico que subyace en el Proyecto Canaima Educativo (PEC), referido al uso del computador como mediador del aprendizaje en el aula. Dicho proyecto fue desarrollado por el Ministerio de Educación de Venezuela, con la finalidad de fortalecer los aprendizajes de los niños y niñas de este país. Los resultados indicaron que el modelo se fundamenta en la teoría del socioconstructivismo; asimismo, propone al alumno como protagonista de su aprendizaje, en una acción colaborativa, interactiva y significativa, y propone al docente como un agente mediador del proceso, que orienta al estudiante sobre la ruta formativa que debe seguir.

El segundo capítulo expone una estrategia de acompañamiento docente para el desarrollo de la competencia en informática educativa de profesores de la Universidad de las Américas en Ecuador, que toma como referente al modelo TPACK para fortalecer los conocimientos tecnológicos y pedagógicos 
de los docentes, con la finalidad de que estos logren transformar sus prácticas, de suerte que respondan a las expectativas de sus estudiantes.

El tercer capítulo, denominado "Estrategias para el mejoramiento del E-Learning en Colombia. El estudio de caso del SENA", realiza una revisión a profundidad del modelo de e-learning a través de las prácticas pedagógicas de más de 300 tutores de los diferentes programas de la institución; asimismo, propone la formulación de diferentes estrategias orientadas a mejorar las actividades y roles de los tutores dentro del proceso formativo de los aprendices.

El cuarto capítulo da cuenta un modelo para la construcción de juegos serios aplicados a la enseñanza-aprendizaje de física cinemática; el docente debe partir de las bases conceptuales del movimiento para plantear los diferentes retos dentro del juego serio, pero resulta más relevante para la formación que los estudiantes formen parte del diseño, pues no solo se identifican con el videojuego desarrollado, sino que aumentan su dedicación y esfuerzo para superar los retos.

Por su parte, el quinto capítulo, denominado "El celular como herramienta educativa para el desarrollo de la competencia digital", presenta una experiencia de aprendizaje móvil implementada en una institución educativa de la Secretaría de Educación de Bogotá. Los principales aportes de este estudio están enfocados a las posibilidades que brinda el uso de dispositivos móviles como herramientas de aprendizaje en la educación básica y media, así como a la necesidad de que el docente asuma un nuevo rol dentro del proceso.

El siguiente capítulo muestra un proyecto desarrollado en la Universidad de La Sabana con el objetivo de identificar los aportes del uso de un mundo virtual en el aprendizaje de las licencias Creative Commons. Los principales resultados del estudio determinaron que la integración de este tipo de tecnología favorece el desarrollo de aprendizajes, debido a que los estudiantes se sienten muy motivados al interactuar en un entorno virtual como Second Life, pero, sobre todo, al poder solucionar los retos planteados en el proyecto.

A continuación, el texto da cuenta de una experiencia de integración de tecnologías educativas para fortalecer el aprendizaje del inglés como lengua extranjera en dos instituciones de educación básica de Cundinamarca. Dentro de sus principales aportes está el uso de un ambiente de aprendizaje mediado por TIC y basado en el aprendizaje entre pares, donde los estudiantes asumen un rol activo en el desarrollo de diálogos que facilitan el aprendizaje de la segunda lengua. 
El capítulo 10 expone una sistematización de experiencias internas desarrolladas en la Universidad Pontificia Bolivariana de Medellín, donde la innovación social se convierte en estrategia pedagógica para la innovación educativa contextualizada. Uno de sus grandes aportes está relacionado con la toma de conciencia por parte de los estudiantes sobre la realidad de su contexto, así como el reconocimiento del compromiso ético que tienen como profesionales en formación con el servicio social.

El siguiente capítulo resume un gran aporte para la enseñanza del área deportiva, al integrar el uso de simuladores digitales en el proceso de enseñanza-aprendizaje de licenciados en ciencias del deporte. Específicamente, este estudio se centró en optimizar el proceso de enseñanza de la recepción del servicio en voleibol, a través de una estrategia que incluía la participación e interacción activa de los estudiantes, la integración de blogs, videos y simuladores. Durante el proceso, los estudiantes mostraron una gran dedicación a mejorar su técnica con base en los datos entregados por el simulador.

Luego, un capítulo que aborda una alternativa desarrollada en la Universidad Nacional para enfrentar una de las problemáticas de la educación superior: la alta repetición de las asignaturas entre los estudiantes. Para solucionar esta problemática, la autora propone el uso de MOOCS dotados de una diversidad de ejercicios realizados en laboratorios virtuales gratuitos, que puedan ser tomados por los estudiantes como complemento al proceso de formación. Esta estrategia ha dado a la universidad una solución, pues los estudiantes logran con los simuladores comprender los conceptos complejos alrededor de la teoría de la física.

Para finalizar, el último capítulo da cuenta de los resultados de un estudio realizado por el Observatorio de Tecnología e Innovación Educativa de la Universidad de La Sabana alrededor de la caracterización de la investigación sobre tecnología educativa en Colombia durante los últimos años. Este estudio identifica las zonas geográficas donde se ha desarrollado el ecosistema de investigación en tecnología educativa en el país, así como las zonas en las cuales aún no se han visto los efectos de la integración de tecnología educativa. Asimismo, muestra que la mayoría de las investigaciones llevadas a cabo por los grupos, en términos de población, tienen un alcance de una institución, localidad o, en el mejor de los casos, un alcance regional; por ello, el efecto que sus resultados pueden tener en Colombia es mínimo, pues si bien algunas de ellas generan un gran aporte local, no suelen afectar las prácticas educativas.

En conclusión, este libro resulta un gran instrumento de navegación para docentes e investigadores interesados en comprender la forma en que 
los procesos de enseñanza-aprendizaje pueden fortalecerse a través de la integración de tecnología educativa, pues no solo trata una diversidad de casos en todos los niveles de formación, sino que brinda orientaciones sobre los roles que los diferentes actores del proceso formativo deben asumir para lograr la materialización de escenarios innovadores en la educación.

El libro completo está disponible en https:/ / repository.upb.edu.co/handle/20.500.11912/5337?locale-attribute=en

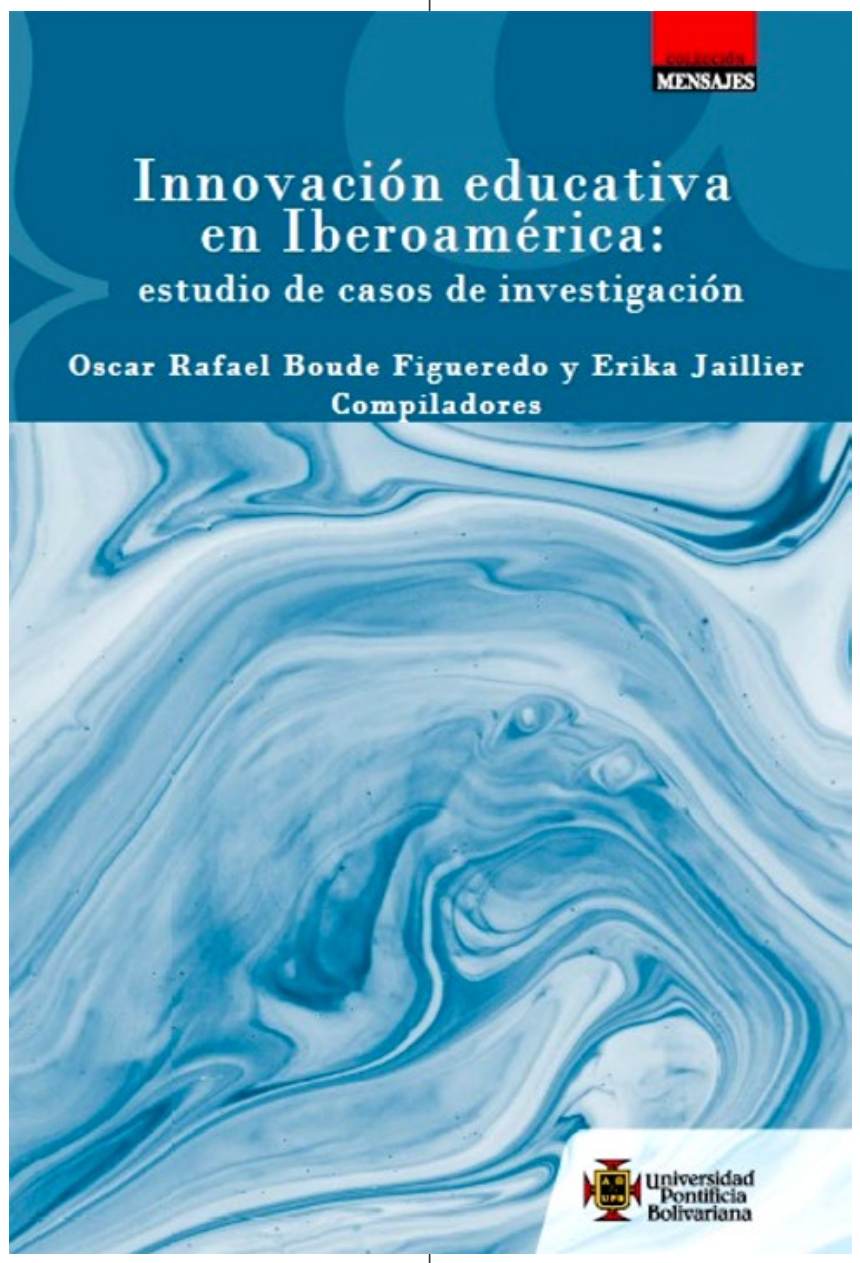

